\title{
PBCAR3/pIRS2-Phosphopeptide-tetanus Peptide Vaccine
}

National Cancer Institute

\section{Source}

National Cancer Institute. pBCAR3/pIRS2-Phosphopeptide-tetanus Peptide Vaccine. NCI Thesaurus. Code C107168.

A vaccine composed of phosphorylated peptides from the tumor associated antigens breast cancer anti-estrogen resistance-3 (BCAR3) and insulin receptor substrate-2 (IRS2) and a tetanus-derived peptide, with potential immunomodulating and antineoplastic activities. Upon administration of pBCAR3/pIRS2 phosphopeptide-tetanus peptide vaccine, the $\mathrm{pBCAR3/pIRS2}$ phosphopeptide may stimulate the immune system to mount a cytotoxic T-lymphocyte $(\mathrm{CT} L)$ response against tumor cells expressing either phosphopeptide. The tetanus peptide serves as an immunoadjuvant and induces a helper T-cell response which may help stimulate an immune response against PBCAR3 and pIRS2-expressing melanoma tumor cells. BCAR3 and IRS2 are upregulated in a variety of cancer cells. 